\title{
Article \\ Clinical Limitations of Tissue Annexin A2 Level as a Predictor of Postoperative Overall Survival in Patients with Hepatocellular Carcinoma
}

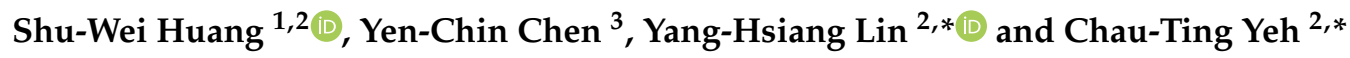 \\ 1 Department of Gastroenterology and Hepatology, New Taipei Municipal Tucheng Hospital, \\ New Taipei 236, Taiwan; huangshuwei@gmail.com \\ 2 Liver Research Center, Chang Gung Memorial Hospital, Linkou, Taoyuan 333, Taiwan \\ 3 Graduate Institute of Clinical Medicine, Chang Gung University, Taoyuan 333, Taiwan; \\ sunnychen168@gmail.com \\ * Correspondence: yhlin0621@cgmh.org.tw (Y.-H.L.); chautingy@gmail.com (C.-T.Y.); \\ Tel.: +886-3328-1200 (ext. 7785) (Y.-H.L.); +886-3328-1200 (ext. 8129) (C.-T.Y.); Fax: +886-3328-2824 (C.-T.Y.)
}

Citation: Huang, S.-W.; Chen, Y.-C.; Lin, Y.-H.; Yeh, C.-T. Clinical

Limitations of Tissue Annexin A2 Level as a Predictor of Postoperative Overall Survival in Patients with Hepatocellular Carcinoma. J. Clin. Med. 2021, 10, 4158. https://doi.org/ $10.3390 /$ jcm10184158

Academic Editor: Ugo Grossi

Received: 12 August 2021

Accepted: 13 September 2021

Published: 15 September 2021

Publisher's Note: MDPI stays neutral with regard to jurisdictional claims in published maps and institutional affiliations.

Copyright: (c) 2021 by the authors. Licensee MDPI, Basel, Switzerland. This article is an open access article distributed under the terms and conditions of the Creative Commons Attribution (CC BY) license (https:// creativecommons.org/licenses/by/ $4.0 /)$.

\begin{abstract}
Hepatocellular carcinoma (HCC) is the second common cause of cancer-related death in Taiwan. Tumor recurrence is frequently observed in HCC patients receiving surgical resection, resulting in unsatisfactory overall survival (OS). Therefore, it is pivotal to identify effective prognostic makers, so that intensive surveillance or adjuvant treatments can be applied to predictively unfavorable patients. Previous studies indicated that Annexin A2 (ANXA2) was an effective prognostic marker in several cancers, including HCC. However, the prognostic value of ANXA2 in Taiwanese HCC patients remains unclear, where a great proportion of patients had chronic hepatitis B with liver cirrhosis. Here, ANXA2 was highly expressed in HCC tissues compared with para-neoplastic noncancerous tissues. Furthermore, high ANXA2 expression in HCC tissues independently predicted shorter OS. In subgroup analysis, however, ANXA2 expression could not effectively predict OS in the following subgroups: female, age $>65$ years old, Child-Pugh classification B, hepatitis B virus surface antigen negative or anti-hepatitis $C$ antibody positive, alcoholism, tumor number $>1$, presence of micro- or macrovascular invasion, absence of capsule, non-cirrhosis and high alpha-fetoprotein. In conclusion, ANXA2 expression in HCC tissues could predict postoperative OS. However, the predictive value was limited in patients with specific clinical conditions.
\end{abstract}

Keywords: hepatocellular carcinoma; annexin A2; prognostic marker; survival outcome

\section{Introduction}

Hepatocellular carcinoma (HCC) is the second most common cause of cancer-related death in Taiwan [1]. Infection with hepatitis B virus (HBV) and hepatitis C virus (HCV) can lead to chronic hepatitis, liver fibrosis, cirrhosis and eventually HCC [2]. Despite the improvement in the treatment of chronic viral hepatitis and the successful implantation of neonatal vaccination program against HBV, HCC is still a severe public health concern in Taiwan [3]. Surgical treatment is considered one of the most efficient therapies for earlystage HCC. However, incidence of tumor recurrence and distant metastasis remains high in HCC patients receiving surgical resection, resulting in unsatisfactory clinical outcomes. Several biomarkers such as alpha-fetoprotein (AFP) were used for diagnosis and outcome prediction in HCC patients. However, approximately $40 \%$ HCC patients still presented with normal levels of AFP, suggesting that the diagnostic and prognostic role of AFP in HCC patients is still limited $[4,5]$. Therefore, it is very important to identify new prognostic makers for these patients, so that more intensive surveillance and/or adjuvant treatments, if available, could be applied to unfavorable patients.

Annexin A2 (ANXA2) belongs to annexin family and is responsible for regulating cell growth, cell-cell junctions and apoptosis [6-8]. ANXA2 has been reported to act as an early- 
stage HCC biomarker [9]. Another study [10] reported that ANXA2 was overexpressed in hepatoma cells compared to normal cells. Depletion of ANXA2 repressed cell proliferation and enhanced 5-fluorouracil-mediated effects via suppression of $\beta$-catenin and cyclin D1 expression. Yang et al. [11] demonstrated that ANXA2 enhanced liver fibrosis through regulation of the von Willebrand factor ( $\mathrm{vWF}$ ) in vitro and in vivo. These findings suggest that ANXA2 plays an oncogenic role in HCC progression. However, another study [12] indicated that expression levels of ANXA2 in HCC tissue and serum specimens were not correlated well with clinical outcomes, suggesting that ANXA2 was not a good prognostic maker for HCC patients with HBV-related liver cirrhosis. Accordingly, the predictive value of ANXA2 in Taiwanese HCC patients needed to be determined, where a great proportion of HCC was HBV-related, arising from a cirrhotic background.

In this study, ANXA2 expression levels were determined by Western blot followed by densitometry-based quantification. The clinical correlation between ANXA2 expression and postoperative outcomes was analyzed in Taiwanese HCC patients.

\section{Materials and Methods}

\subsection{Patients and Basic Clinical Data}

This was a retrospective longitudinal cohort study. From 1996 to 2006, a total of 148 paired HCC specimens (cancerous and para-neoplastic noncancerous tissues) obtained from surgical resection of HCC in LinKou Chang Gung Memorial Hospital were retrieved (cohort 1) and subjected to ANXA2 expression analysis by Western blot. Samples providing sufficient amounts of protein for Western blot analysis were randomly selected from the tissue bank. Only those with written informed consent from patients were included. The clinicopathological data were collected, including age, gender, tumor number, tumor size, histological grading, microvascular invasion, macrovascular invasion, capsule, microsatellite distribution, liver cirrhosis, Child-Pugh classification of liver function, ascites, alpha-fetoprotein (AFP), albumin, bilirubin, prothrombin time (PT), aspartate transaminase (AST), alanine transaminase (ALT), HBV surface antigen (HBsAg), anti-HCV antibody and alcoholism (Table 1). Meanwhile, longitudinal data of recurrence-free survival (RFS) and overall survival (OS) were collected and calculated for survival outcome analysis. RFS was calculated as the period from the time of operation to the time of tumor recurrence or metastasis. OS was calculated as the period from the time of operation to the time of death. The time-point when a patient was lost to follow up was censored. In addition, expression levels of ANXA2 in online available datasets (TCGA, cohort 2 and GSE14520, (cohort 3) were analyzed to further confirm its prognostic value in patients with HCC [13].

\subsection{Western Blot Analysis}

The procedure of Western blot analysis was described in the previous study [14]. Cells were collected and lysed with RIPA buffer (BIOTOOLS Co., Ltd., Taipei, Taiwan, TAARZBZ5) containing protease inhibitors (Merck Millipore, Temecula, CA, USA, \#539134). Protein concentrations of these samples were determined using the Bradford assay. Protein samples $(60 \mu \mathrm{g})$ were loaded and separated by SDS-PAGE. The voltage (V) at stacking gel and resolution gel was $60-80$ and $120-150 \mathrm{~V}$, respectively. After loading dye reached the end of the gel, the gel was transferred to $0.45 \mu \mathrm{m}$ PVDF membrane. The blocking buffer was added to the membrane for $1 \mathrm{~h}$ at room temperature. The membrane was incubated with specific antibody against ANXA2 (BD Biosciences, Franklin Lakes, NJ, USA) overnight at $4{ }^{\circ} \mathrm{C}$. In addition, $\beta$-actin (Sigma-Aldrich, St Louis, MO, USA) was also visualized and used as loading control. The signal intensity of ANXA2 and $\beta$-actin was calculated by Image Gauge software (Fujifilm, Tokyo, Japan). 
Table 1. Basic clinicopathological factors of patients with or without liver cirrhosis (cohort 1).

\begin{tabular}{|c|c|c|c|c|}
\hline Variable & All Patients & Non-Cirrhosis & Cirrhosis & $p$ \\
\hline Patient number & 148 & 80 & 68 & \\
\hline $\begin{array}{l}\text { Gender } \\
\text { Female } \\
\text { Male }\end{array}$ & $\begin{array}{c}36(24.3 \%) \\
112(75.7 \%)\end{array}$ & $\begin{array}{l}20(25.0 \%) \\
60(75.0 \%)\end{array}$ & $\begin{array}{l}16(23.5 \%) \\
52(76.5 \%)\end{array}$ & 0.835 \\
\hline Age (years) & $56.0 \pm 14.9$ & $54.2 \pm 16.1$ & $58.1 \pm 13.2$ & 0.106 \\
\hline $\begin{array}{c}\text { Child-Pugh Classification } \\
\text { A } \\
\text { B } \\
\end{array}$ & $\begin{array}{c}127(85.8 \%) \\
21(14.2 \%)\end{array}$ & $\begin{array}{l}70(87.5 \%) \\
10(12.5 \%)\end{array}$ & $\begin{array}{l}57(83.8 \%) \\
11(16.2 \%)\end{array}$ & 0.523 \\
\hline $\begin{array}{l}\text { Ascites } \\
\text { No } \\
\text { Yes }\end{array}$ & $\begin{array}{c}136(91.9 \%) \\
12(8.1 \%)\end{array}$ & $\begin{array}{c}74(92.5 \%) \\
6(7.5 \%)\end{array}$ & $\begin{array}{c}62(91.2 \%) \\
6(8.8 \%)\end{array}$ & 0.769 \\
\hline $\begin{array}{l}\text { HBsAg } \\
\text { Negative } \\
\text { Positive } \\
\end{array}$ & $\begin{array}{c}46(31.1 \%) \\
102(68.9 \%)\end{array}$ & $\begin{array}{l}24(30.0 \%) \\
56(70.0 \%)\end{array}$ & $\begin{array}{l}22(32.4 \%) \\
46(67.6 \%)\end{array}$ & 0.758 \\
\hline $\begin{array}{l}\text { Anti-HCV Ab } \\
\text { Negative } \\
\text { Positive } \\
\end{array}$ & $\begin{array}{c}107(72.3 \%) \\
41(27.7 \%)\end{array}$ & $\begin{array}{l}68(85.0 \%) \\
12(15.0 \%)\end{array}$ & $\begin{array}{l}39(57.4 \%) \\
29(42.6 \%)\end{array}$ & $<0.001$ * \\
\hline $\begin{array}{c}\text { Alcohol consumption } \\
\text { No } \\
\text { Yes } \\
\end{array}$ & $\begin{array}{c}104(70.3 \%) \\
44(29.7 \%)\end{array}$ & $\begin{array}{l}59(73.8 \%) \\
21(26.3 \%)\end{array}$ & $\begin{array}{l}45(66.2 \%) \\
23(33.8 \%)\end{array}$ & 0.315 \\
\hline Tumor status & & & & \\
\hline $\begin{array}{c}\text { Tumor number } \\
1 \\
\geq 2 \\
\end{array}$ & $\begin{array}{l}95(64.2 \%) \\
53(35.8 \%)\end{array}$ & $\begin{array}{l}51(63.8 \%) \\
29(36.3 \%)\end{array}$ & $\begin{array}{l}44(64.7 \%) \\
24(35.3 \%)\end{array}$ & 0.904 \\
\hline Tumor size $(\mathrm{cm})$ & $6.9 \pm 4.8$ & $8.5 \pm 5.1$ & $5.1 \pm 3.6$ & $<0.001$ * \\
\hline $\begin{array}{c}\text { Histological grading } \\
1-2 \\
3-4\end{array}$ & $\begin{array}{c}47(31.8 \%) \\
101(68.2 \%)\end{array}$ & $\begin{array}{l}22(27.5 \%) \\
58(72.5 \%)\end{array}$ & $\begin{array}{l}25(36.8 \%) \\
43(63.2 \%)\end{array}$ & 0.228 \\
\hline $\begin{array}{c}\text { Microvascular invasion } \\
\text { No } \\
\text { Yes } \\
\end{array}$ & $\begin{array}{c}103(69.6 \%) \\
45(30.4 \%)\end{array}$ & $\begin{array}{l}54(67.5 \%) \\
26(32.5 \%)\end{array}$ & $\begin{array}{l}49(72.1 \%) \\
19(27.9 \%)\end{array}$ & 0.548 \\
\hline $\begin{array}{c}\text { Macrovascular invasion } \\
\text { No } \\
\text { Yes } \\
\end{array}$ & $\begin{array}{c}136(91.9 \%) \\
12(8.1 \%)\end{array}$ & $\begin{array}{c}74(92.5 \%) \\
6(7.5 \%)\end{array}$ & $\begin{array}{c}62(91.2 \%) \\
6(8.8 \%)\end{array}$ & 0.769 \\
\hline $\begin{array}{c}\text { Capsule } \\
\text { No } \\
\text { Yes } \\
\end{array}$ & $\begin{array}{c}37(25.0 \%) \\
111(75.0 \%)\end{array}$ & $\begin{array}{l}22(27.5 \%) \\
58(72.5 \%)\end{array}$ & $\begin{array}{l}15(22.1 \%) \\
53(77.9 \%)\end{array}$ & 0.446 \\
\hline $\begin{array}{c}\text { Microsatellite distribution } \\
\text { No } \\
\text { Yes }\end{array}$ & $\begin{array}{c}123(83.1 \%) \\
25(16.9 \%)\end{array}$ & $\begin{array}{l}63(78.8 \%) \\
17(21.3 \%)\end{array}$ & $\begin{array}{c}60(88.2 \%) \\
8(11.8 \%)\end{array}$ & 0.125 \\
\hline $\begin{array}{c}\text { Annexin A2 expression } \\
<0.8 \text { (Low) } \\
\geq 0.8 \text { (High) }\end{array}$ & $\begin{array}{l}96(64.9 \%) \\
52(35.1 \%)\end{array}$ & $\begin{array}{l}62(77.5 \%) \\
18(22.5 \%)\end{array}$ & $\begin{array}{l}34(50.0 \%) \\
34(50.0 \%)\end{array}$ & $<0.001$ * \\
\hline Laboratory data & & & & \\
\hline $\operatorname{AFP}(n g / m L)$ & $14.9(1.5-327.500)$ & $6.5(2.9-327.500)$ & $22.0(1.5-89,637.7)$ & 0.642 \\
\hline Albumin (g/dL) & $3.7 \pm 0.7$ & $3.8 \pm 0.7$ & $3.7 \pm 0.6$ & 0.427 \\
\hline Bilirubin (mg/dL) & $1.3 \pm 1.7$ & $1.4 \pm 2.1$ & $1.2 \pm 1.0$ & 0.550 \\
\hline Prothrombin time (sec) & $12.4 \pm 1.5$ & $12.1 \pm 1.5$ & $12.7 \pm 1.5$ & $0.042 *$ \\
\hline AST (U/L) & $94.6 \pm 118.8$ & $111.7 \pm 151.0$ & $74.5 \pm 57.7$ & 0.044 * \\
\hline $\operatorname{ALT}(\mathrm{U} / \mathrm{L})$ & $77.0 \pm 96.8$ & $87.9 \pm 120.5$ & $64.2 \pm 56.0$ & 0.119 \\
\hline Creatinine (mg/dL) & $1.2 \pm 1.4$ & $1.3 \pm 1.8$ & $1.1 \pm 0.7$ & 0.428 \\
\hline
\end{tabular}

Abbreviations: AST, aspartate aminotransferase; ALT, alanine aminotransferase; AFP, $\alpha$-fetoprotein; HBsAg, hepatitis B surface antigen;

Anti-HCV Ab, anti-hepatitis $\mathrm{C}$ virus antibody. ${ }^{*} p<0.05$. 


\subsection{Statistical Analysis}

The univariate analysis, multivariate analysis, Kaplan-Meier survival curve and forest plot analysis were performed using SPSS version 20 (SPSS Inc., Chicago, IL, USA). $p$ values $<0.05$ were considered significant $\left({ }^{*} p<0.05\right)$.

\section{Results}

\subsection{Elevated ANXA2 Expression Is Negatively Correlated with Clinical Outcomes}

A total of 148 HCC patients receiving surgical resection were included. Of them, $80(54 \%)$ patients were non-cirrhotic, and $68(46 \%)$ of patients were cirrhotic. The basic clinical data were listed in Table 1. Compared with the non-cirrhosis group, the liver cirrhosis group had higher anti-HCV-positive rate, smaller tumor size, higher proportion of high ANXA2 expression, longer PT prolongation and lower AST level. To investigate whether ANXA2 acted as a prognostic biomarker, the expression levels of ANXA2 in HCC specimens were determined by Western blot followed by densitometry semi-quantification. The cancerous to non-cancerous $(\mathrm{T} / \mathrm{N})$ ratios of ANXA2 were calculated and the minimal $p$ value method was applied to determine the cut off [15]. We found that ANXA2 expression was highly expressed in HCC tissues compared to noncancerous tissues (Figure 1A, $p<0.001)$. We retrieved the longitudinal data of RFS and OS to analyze whether AXNA2 expression (calculated as $\mathrm{T} / \mathrm{N}$ ratio) was associated with prognosis in HCC. Kaplan-Meier plot with log-rank analysis showed that there was no significant association between AXNA2 expression (the T/N ratio) and RFS $(p>0.05)$. However, patients with high ANXA2 expression ( $\mathrm{T} / \mathrm{N}$ ratio $\geq 0.8$ ) had a significantly shorter OS compared to those with low ANXA2 expression (Figure 1B). Similar results were observed in datasets available online (TCGA, cohort 2 and GSE14520, cohort 3) (Figure S1A,B). These findings clearly support that ANXA2 acts as a prognostic maker in patients with HCC. Notably, ANXA2 expression was positively correlated with cirrhosis, AST, anti-HCV antibody and the presence of capsule (Table 2). Taken together, ANXA2 might serve as a prognostic factor for HCC patients receiving surgical treatment.

A

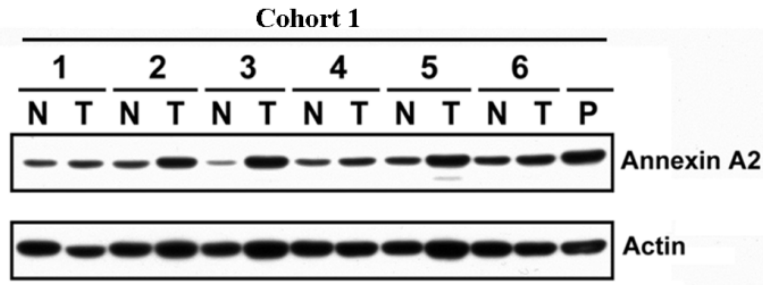

B

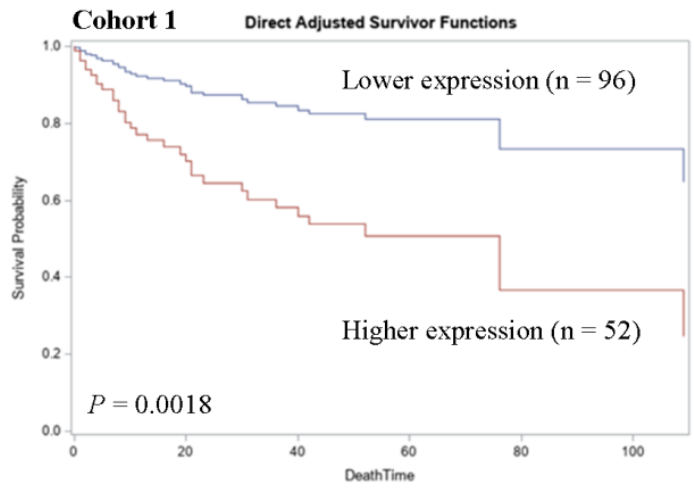

Figure 1. ANXA2 is clinically relevant in HCC. (A) Protein levels of ANXA2 in tumor tissues (T) and non-tumor tissues (N) were determined by Western blotting. P: positive control. (B) Kaplan-Meier survival curves with log-rank test stratified by high ANXA2 expression (higher $\mathrm{T} / \mathrm{N}$ ratio $\geq 0.8$ ) and low ANXA2 expression. 
Table 2. Clinicopathological correlations of ANXA2 in HCC specimens (cohort 1).

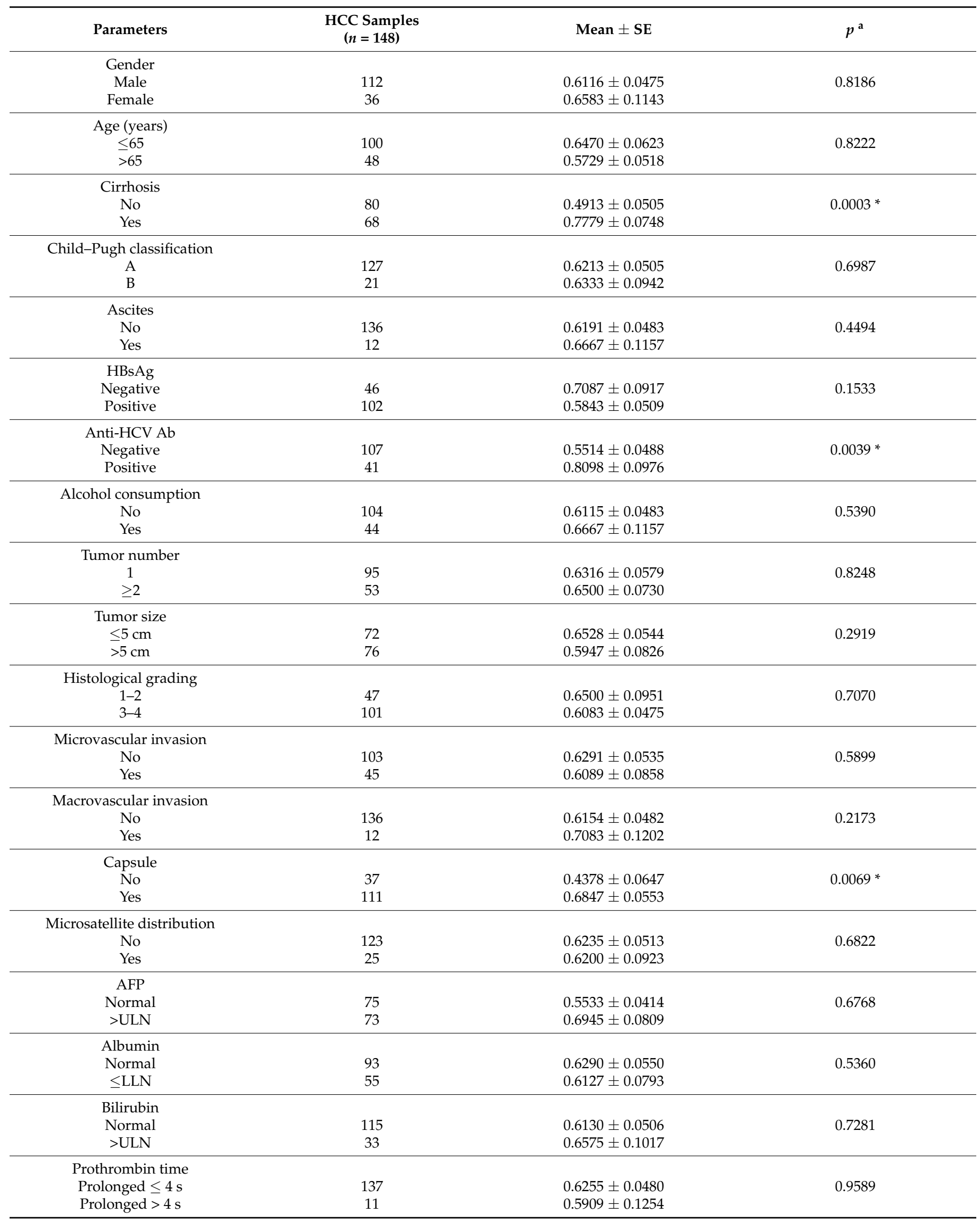


Table 2. Cont.

\begin{tabular}{cccc}
\hline Parameters & $\begin{array}{c}\text { HCC Samples } \\
(\boldsymbol{n}=\mathbf{1 4 8})\end{array}$ & Mean \pm SE & $p^{\mathbf{a}}$ \\
\hline AST & 45 & $0.4311 \pm 0.0490$ & $0.0048^{*}$ \\
Normal & 103 & $0.7068 \pm 0.0597$ & 0.2407 \\
>ULN & 60 & $0.5800 \pm 0.0742$ & $0.6523 \pm 0.0571$ \\
ALT & 88 & & 0.3514 \\
Normal & & & \\
>ULN & 117 & $0.5820 \pm 0.0424$ & \\
\hline Creatinine & 31 & 0.1438 & \\
Normal & & & \\
\hline ULN &
\end{tabular}

${ }^{a}$ : Mann-Whitney U test (for two groups). ${ }^{*} p<0.05$. Abbreviations: SE, standard error; ULN, upper limit of normal; LLN, lower limit of normal; AST, aspartate aminotransferase; ALT, alanine aminotransferase; AFP, $\alpha$-fetoprotein; HBsAg, hepatitis B surface antigen; Anti-HCV $\mathrm{Ab}$, anti-hepatitis $\mathrm{C}$ virus antibody.

\subsection{Clinicopathological Predictors for RFS and OS}

To identify the clinicopathological predictors for RFS and OS, univariate and multivariate Cox proportional hazard analysis was performed and is shown in Tables 3 and 4 . For RFS, presence of ascites, tumor number $\geq 2$, presence of microvascular invasion and microsatellite distribution of tumors, high Annexin A2 expression, AFP and AST > upper limit of normal were associated with RFS by univariate analysis. Multivariate analysis showed that the presence of ascites, tumor number $\geq 2$ and AST $>$ upper limit of normal were the independent predictors for RFS (Table 3). For OS, age $>65$ years, Child-Pugh liver function classification $B$, the presence of ascites, microvascular invasion, high ANXA2 expression, AFP, bilirubin and AST > upper limit of normal were associated with short OS in the univariate Cox proportional analysis. Multivariate analysis showed that Child-Pugh liver function classification B, presence of ascites and high AXNA2 expression were the independent predictors for OS (Table 4).

Table 3. Analysis of factors that influenced RFS of all patients (cohort 1).

\begin{tabular}{|c|c|c|c|c|c|c|c|}
\hline \multirow[b]{3}{*}{ Parameters } & \multirow[b]{3}{*}{$n$} & \multicolumn{6}{|c|}{ RFS } \\
\hline & & \multicolumn{3}{|c|}{ Univariate Analysis } & \multicolumn{3}{|c|}{ Multivariate Analysis } \\
\hline & & HR & $95 \% \mathrm{CI}$ & $p$ & HR & $95 \% \mathrm{CI}$ & $p$ \\
\hline \multicolumn{8}{|l|}{ Gender } \\
\hline Female & 36 & & & & & & \\
\hline Male & 112 & 1.263 & $0.760-2.098$ & 0.3668 & & & \\
\hline \multicolumn{8}{|l|}{ Age (years) } \\
\hline$\leq 65$ & 100 & & & & & & \\
\hline$>65$ & 48 & 0.785 & $0.491-1.256$ & 0.3125 & & & \\
\hline \multicolumn{8}{|l|}{ Cirrhosis } \\
\hline No & 80 & & & & & & \\
\hline Yes & 68 & 1.466 & $0.967-2.223$ & 0.0717 & & & \\
\hline \multicolumn{8}{|l|}{$\begin{array}{l}\text { Child-Pugh } \\
\text { classification }\end{array}$} \\
\hline A & 127 & & & & & & \\
\hline B & 21 & 1.345 & $0.714-2.535$ & 0.3587 & & & \\
\hline \multicolumn{8}{|l|}{ Ascites } \\
\hline No & 136 & & & & & & \\
\hline Yes & 12 & 3.301 & $1.715-6.352$ & $<0.001 *$ & 2.274 & $1.156-4.472$ & $0.0173 *$ \\
\hline \multicolumn{8}{|l|}{ HBsAg } \\
\hline Negative & 46 & & & & & & \\
\hline Positive & 102 & 1.113 & $0.701-1.767$ & 0.6507 & & & \\
\hline \multicolumn{8}{|l|}{ Anti-HCV Ab } \\
\hline Negative & 107 & & & & & & \\
\hline Positive & 41 & 1.180 & $0.736-1.891$ & 0.4923 & & & \\
\hline \multicolumn{8}{|l|}{$\begin{array}{c}\text { Alcohol } \\
\text { consumption }\end{array}$} \\
\hline No & 104 & & & & & & \\
\hline Yes & 44 & 1.205 & $0.775-1.873$ & 0.4069 & & & \\
\hline
\end{tabular}


Table 3. Cont.

\begin{tabular}{|c|c|c|c|c|c|c|c|}
\hline \multirow[b]{3}{*}{ Parameters } & \multirow[b]{3}{*}{$n$} & \multicolumn{6}{|c|}{ RFS } \\
\hline & & \multicolumn{3}{|c|}{ Univariate Analysis } & \multicolumn{3}{|c|}{ Multivariate Analysis } \\
\hline & & HR & $95 \%$ CI & $p$ & HR & $95 \%$ CI & $p$ \\
\hline \multicolumn{8}{|l|}{ Tumor status } \\
\hline \multicolumn{8}{|l|}{ Tumor number } \\
\hline 1 & 95 & & & & & & \\
\hline$\geq 2$ & 53 & 3.240 & $2.097-5.005$ & $<0.0001^{*}$ & 2.649 & $1.571-4.467$ & 0.0003 * \\
\hline \multicolumn{8}{|l|}{ Tumor size $(\mathrm{cm})$} \\
\hline$\leq 5$ & 72 & & & & & & \\
\hline$>5$ & 76 & 1.475 & $0.968-2.247$ & 0.0706 & & & \\
\hline \multicolumn{8}{|l|}{$\begin{array}{l}\text { Histological } \\
\text { grading }\end{array}$} \\
\hline $1-2$ & 47 & & & & & & \\
\hline $3-4$ & 101 & 1.217 & $0.760-1.950$ & 0.4133 & & & \\
\hline \multicolumn{8}{|l|}{$\begin{array}{l}\text { Microvascular } \\
\text { invasion }\end{array}$} \\
\hline No & 103 & & & & & & \\
\hline Yes & 45 & 2.514 & $1.623-3.895$ & $<0.0001^{*}$ & 1.489 & $0.878-2.523$ & 0.1394 \\
\hline \multirow{2}{*}{\multicolumn{8}{|c|}{$\begin{array}{l}\text { Macrovascular } \\
\text { invasion } \\
\text { thrombosis }\end{array}$}} \\
\hline & & & & & & & \\
\hline No & 136 & & & & & & \\
\hline Yes & 12 & 1.516 & $0.760-3.028$ & 0.2379 & & & \\
\hline \multicolumn{8}{|l|}{ Capsule } \\
\hline No & 37 & & & & & & \\
\hline Yes & 111 & 0.778 & $0.486-1.245$ & 0.2954 & & & \\
\hline \multicolumn{8}{|l|}{$\begin{array}{l}\text { Microsatellite } \\
\text { distribution }\end{array}$} \\
\hline No & 123 & & & & & & \\
\hline Yes & 25 & 2.300 & $1.391-3.803$ & $0.0012 *$ & 0.881 & $0.468-1.661$ & 0.6962 \\
\hline \multicolumn{8}{|l|}{$\begin{array}{l}\text { Annexin A2 } \\
\text { expression }\end{array}$} \\
\hline$<0.8$ (Low) & 96 & & & & & & \\
\hline$\geq 0.8$ (High) & 52 & 1.726 & $1.120-2.659$ & 0.0133 * & 1.459 & $0.934-2.279$ & 0.0969 \\
\hline \multicolumn{8}{|l|}{ Laboratory data } \\
\hline \multicolumn{8}{|l|}{ AFP } \\
\hline Normal & 75 & & & & & & \\
\hline >ULN & 73 & 1.903 & $1.248-2.900$ & $0.0028 *$ & 1.544 & 0.994-2.399 & 0.0531 \\
\hline \multicolumn{8}{|l|}{ Albumin } \\
\hline Normal & 93 & 0.769 & $0.497-1.189$ & 0.2369 & & & \\
\hline$\leq \mathrm{LLN}$ & 55 & & & & & & \\
\hline \multicolumn{8}{|l|}{ Bilirubin } \\
\hline Normal & 115 & & & & & & \\
\hline$>\mathrm{ULN}$ & 33 & 1.384 & $0.847-2.261$ & 0.1951 & & & \\
\hline \multicolumn{8}{|l|}{$\begin{array}{l}\text { Prothrombin } \\
\text { time }\end{array}$} \\
\hline Prolonged $\leq 4 \mathrm{~s}$ & 137 & & & & & & \\
\hline Prolonged $>4 \mathrm{~s}$ & 11 & 1.289 & $0.619-2.686$ & 0.4981 & & & \\
\hline \multicolumn{8}{|l|}{ AST } \\
\hline Normal & 45 & & & & & & \\
\hline$>\mathrm{ULN}$ & 103 & 1.846 & $1.132-3.010$ & 0.0141 * & 1.719 & $1.027-2.880$ & 0.0394 * \\
\hline ALT & & & & & & & \\
\hline Normal & 60 & & & & & & \\
\hline$>\mathrm{ULN}$ & 88 & 1.304 & $0.852-1.995$ & 0.2210 & & & \\
\hline Creatinine & & & & & & & \\
\hline Normal & 117 & & & & & & \\
\hline$>\mathrm{ULN}$ & 31 & 0.898 & $0.515-1.568$ & 0.7057 & & & \\
\hline
\end{tabular}

${ }^{*} p<0.05$. Abbreviations: RFS, recurrence-free survival; HR, hazard ratio; CI, confidence interval; ULN, upper limit of normal; LLN, lower limit of normal; AST, aspartate aminotransferase; ALT, alanine aminotransferase; AFP, $\alpha$-fetoprotein; HBsAg, hepatitis B surface antigen; Anti-HCV Ab, anti-hepatitis C virus antibody. 
Table 4. Analysis of factors that influenced OS of all patients (cohort 1).

\begin{tabular}{|c|c|c|c|c|c|c|c|}
\hline \multirow[b]{3}{*}{ Parameters } & \multirow[b]{3}{*}{$n$} & \multicolumn{6}{|c|}{ OS } \\
\hline & & \multicolumn{3}{|c|}{ Univariate Analysis } & \multicolumn{3}{|c|}{ Multivariate Analysis } \\
\hline & & HR & $95 \% \mathrm{CI}$ & $p$ & HR & $95 \% \mathrm{CI}$ & $p$ \\
\hline \multicolumn{8}{|l|}{ Gender } \\
\hline Female & 36 & & & & & & \\
\hline Male & 112 & 1.826 & $0.701-4.755$ & 0.2175 & & & \\
\hline \multicolumn{8}{|l|}{ Age (years) } \\
\hline$\leq 65$ & 100 & & & & & & \\
\hline$>65$ & 48 & 0.345 & $0.121-0.986$ & 0.0470 * & 0.494 & $0.160-1.527$ & 0.2204 \\
\hline \multicolumn{8}{|l|}{ Cirrhosis } \\
\hline No & 80 & & & & & & \\
\hline Yes & 68 & 1.357 & $0.677-2.720$ & 0.3891 & & & \\
\hline \multicolumn{8}{|l|}{$\begin{array}{l}\text { Child-Pugh } \\
\text { classification }\end{array}$} \\
\hline A & 127 & & & & & & \\
\hline B & 21 & 4.894 & $2.345-10.215$ & $<0.001 *$ & 3.687 & $1.484-9.159$ & $0.0050 *$ \\
\hline \multicolumn{8}{|l|}{ Ascites } \\
\hline No & 136 & & & & & & \\
\hline Yes & 12 & 4.241 & $1.812-9.926$ & $<0.001 *$ & 3.361 & $1.328-8.507$ & 0.0105 * \\
\hline \multicolumn{8}{|l|}{ HBsAg } \\
\hline Negative & 46 & & & & & & \\
\hline Positive & 102 & 1.003 & $0.463-2.171$ & 0.9949 & & & \\
\hline \multicolumn{8}{|l|}{ Anti-HCV Ab } \\
\hline Negative & 107 & & & & & & \\
\hline Positive & 41 & 1.026 & $0.460-2.287$ & 0.9509 & & & \\
\hline \multicolumn{8}{|l|}{$\begin{array}{c}\text { Alcohol } \\
\text { consumption }\end{array}$} \\
\hline No & 104 & & & & & & \\
\hline Yes & 44 & 1.723 & $0.856-3.468$ & 0.1275 & & & \\
\hline \multicolumn{8}{|l|}{ Tumor status } \\
\hline \multicolumn{8}{|l|}{ Tumor number } \\
\hline 1 & 95 & & & & & & \\
\hline$\geq 2$ & 53 & 1.666 & $0.807-3.439$ & 0.1679 & & & \\
\hline \multicolumn{8}{|l|}{ Tumor size $(\mathrm{cm})$} \\
\hline$\leq 5$ & 72 & & & & & & \\
\hline$>5$ & 76 & 1.731 & $0.834-3.592$ & 0.1410 & & & \\
\hline \multicolumn{8}{|l|}{$\begin{array}{l}\text { Histological } \\
\text { grading }\end{array}$} \\
\hline $1-2$ & 47 & & & & & & \\
\hline $3-4$ & 101 & 1.108 & $0.506-2.429$ & 0.7974 & & & \\
\hline \multicolumn{8}{|l|}{ Microvascular } \\
\hline Invasion & 103 & & & & & & \\
\hline $\begin{array}{l}\text { No } \\
\text { Yes }\end{array}$ & 45 & 2.796 & $1.356-5.765$ & $0.0053 *$ & 1.921 & $0.873-4.227$ & 0.1045 \\
\hline $\begin{array}{c}\text { Macrovascula } \\
\text { invasion } \\
\text { thrombosis }\end{array}$ & & & & & & & \\
\hline No & 136 & & & & & & \\
\hline Yes & 12 & 2.488 & $0.953-6.495$ & 0.0627 & & & \\
\hline Capsule & & & & & & & \\
\hline No & 37 & & & & & & \\
\hline Yes & 111 & 0.811 & $0.363-1.811$ & 0.6097 & & & \\
\hline $\begin{array}{l}\text { Microsatellite } \\
\text { distribution }\end{array}$ & & & & & & & \\
\hline No & 123 & & & & & & \\
\hline Yes & 25 & 2.172 & $0.964-4.893$ & 0.0612 & & & \\
\hline $\begin{array}{l}\text { Annexin A2 } \\
\text { expression } \\
<0.8 \text { (Low) }\end{array}$ & & & & & & & \\
\hline $\begin{array}{l}<0.8 \text { (Low) } \\
\geq 0.8 \text { (High) }\end{array}$ & $\begin{array}{l}96 \\
52\end{array}$ & 3.210 & $1.542-6.684$ & $0.0018 *$ & 2.497 & $1.109-5.619$ & 0.0270 * \\
\hline Laboratory dat & & & & & & & \\
\hline AFP & & & & & & & \\
\hline Normal & 75 & & & & & & \\
\hline$>$ ULN & 73 & 2.292 & $1.102-4.766$ & $0.0264 *$ & 1.381 & $0.603-3.162$ & 0.4446 \\
\hline
\end{tabular}


Table 4. Cont.

\begin{tabular}{|c|c|c|c|c|c|c|c|}
\hline \multirow[b]{3}{*}{ Parameters } & \multirow[b]{3}{*}{$n$} & \multicolumn{6}{|c|}{ os } \\
\hline & & \multicolumn{3}{|c|}{ Univariate Analysis } & \multicolumn{3}{|c|}{ Multivariate Analysis } \\
\hline & & HR & $95 \%$ CI & $p$ & HR & $95 \%$ CI & $p$ \\
\hline $\begin{array}{c}\text { Albumin } \\
\text { Normal } \\
\leq \text { LLN }\end{array}$ & $\begin{array}{l}93 \\
55\end{array}$ & 0.515 & $0.257-1.036$ & 0.0626 & & & \\
\hline $\begin{array}{l}\text { Bilirubin } \\
\text { Normal } \\
\text { >ULN }\end{array}$ & $\begin{array}{c}115 \\
33\end{array}$ & 2.186 & $1.033-4.627$ & $0.0410^{*}$ & 1.077 & $0.457-2.538$ & 0.8659 \\
\hline $\begin{array}{c}\text { Prothrombin } \\
\text { time } \\
\text { Prolonged } \leq 4 \mathrm{~s} \\
\text { Prolonged }>4 \mathrm{~s}\end{array}$ & $\begin{array}{c}137 \\
11\end{array}$ & 2.031 & $0.773-5.340$ & 0.1508 & & & \\
\hline $\begin{array}{c}\text { AST } \\
\text { Normal } \\
\text { >ULN }\end{array}$ & $\begin{array}{c}45 \\
103\end{array}$ & 3.362 & $1.179-9.586$ & $0.0233 *$ & 1.955 & $0.630-6.062$ & 0.2458 \\
\hline $\begin{array}{c}\text { ALT } \\
\text { Normal } \\
>\text { ULN }\end{array}$ & $\begin{array}{l}60 \\
88\end{array}$ & 1.063 & $0.524-2.154$ & 0.8662 & & & \\
\hline $\begin{array}{l}\text { Creatinine } \\
\text { Normal } \\
>\text { ULN }\end{array}$ & $\begin{array}{c}117 \\
31\end{array}$ & 0.683 & $0.263-1.776$ & 0.4346 & & & \\
\hline
\end{tabular}

${ }^{*} p<0.05$. Abbreviations: OS, overall survival; HR, hazard ratio; CI, confidence interval; ULN, upper limit of normal; LLN, lower limit of normal; AST, aspartate aminotransferase; ALT, alanine aminotransferase; AFP, $\alpha$-fetoprotein; HBsAg, hepatitis B surface antigen; Anti-HCV $\mathrm{Ab}$, anti-hepatitis $\mathrm{C}$ virus antibody.

\subsection{ANXA2 Expression Levels in HCC Tissues Are an Effective Prognosis Predictor in Specific Clinical Subgroups of HCC}

In addition, we studied the predictive role of high AXNA2 expression in various clinical subgroups using Cox proportional hazard method (Figure 2). The ANXA2 expression was associated with OS when all HCC patients were included for assessment. In addition, it was also associated with OS in the following subgroups: male (HR $=2.772$, $95 \%$ CI 1.254-6.130, $p=0.0118)$, age $\leq 65$ (HR $=2.943,95 \%$ CI 1.361-6.367, $p=0.0061)$, Child-Pugh liver function classification A ( $\mathrm{HR}=3.324,95 \%$ CI 1.354-8.159, $p=0.0087$ ), no ascites $(\mathrm{HR}=2.705,95 \%$ CI 1.182-6.188, $p=0.0185)$, HBsAg-positive (HR $=3.269$, 95\% CI 1.375-7.771, $p=0.0073$ ), anti-HCV Ab negative (HR = 3.796, 95\% CI 1.635-8.813, $p=0.0019)$, no alcohol consumption ( $\mathrm{HR}=3.398,95 \% \mathrm{CI} 1.314-8.787, p=0.0116)$, tumor number $=1(\mathrm{HR}=6.027,95 \%$ CI 2.109-17.223, $p=0.0008)$, tumor size $\leq 5 \mathrm{~cm}(\mathrm{HR}=6.241$, 95\% CI 1.321-29.490, $p=0.0208)$, tumor size $>5 \mathrm{~cm}(\mathrm{HR}=2.860,95 \%$ CI $1.185-6.900$, $p=0.0194)$, histological grading $1-2(\mathrm{HR}=6.057,95 \% \mathrm{CI} 1.211-30.285, p=0.0283)$ and $3-4$ $(\mathrm{HR}=2.592,95 \% \mathrm{CI} 1.116-6.021, p=0.0267)$, no microvascular invasion $(\mathrm{HR}=3.772,95 \%$ CI 1.365-10.422, $p=0.0105)$, no macrovascular invasion (HR $=2.983,95 \%$ CI 1.367-6.506, $p=0.006)$, presence of capsule ( $\mathrm{HR}=3.877,95 \%$ CI 1.649-9.117, $p=0.0019)$, no microsatellite distribution ( $\mathrm{HR}=3.338,95 \% \mathrm{CI} 1.422-7.836, p=0.0056)$, presence of cirrhosis (HR = 5.220, 95\% CI 1.464-18.610, $p=0.0061$ ), normal AFP (HR $=7.756,95 \%$ CI 1.600-37.603, $p=0.011$ ), Albumin $\leq$ LLN (HR $=3.262,95 \%$ CI 1.106-9.623, $p=0.0322$ ), normal bilirubin (HR $=3.585,95 \%$ CI 1.478-8.699, $p=0.0048)$, Prothrombin time $\leq 4 \mathrm{~s}(\mathrm{HR}=3.519,95 \% \mathrm{CI}$ $1.592-7.782, p=0.0019)$, AST $>$ ULN (HR $=3.095,95 \%$ CI 1.373-6.977, $p=0.0064)$, ALT nor$\mathrm{mal}(\mathrm{HR}=4.599,95 \% \mathrm{CI} 1.518-13.933, p=0.007)$ and $>\mathrm{ULN}(\mathrm{HR}=2.841,95 \% \mathrm{CI} 1.045-7.720$, $p=0.0407)$ and creatinine normal $(\mathrm{HR}=3.098,95 \% \mathrm{CI} 1.388-6.915, p=0.0058)$. In contrast, the association was not present in the following subgroups ( $p>0.05$ for all): female, age $>65$ years, Child-Pugh classification B, presence of ascites, HBsAg negative, anti-HCVpositive, alcoholism; tumor number $\geq 2$, micro- or macrovascular invasion, microsatellite distribution of tumors, non-cirrhosis, AFP or bilirubin > upper limit of normal, PT prolongation $>4 \mathrm{~s}$, normal AST or creatinine $>$ upper limit of normal. 


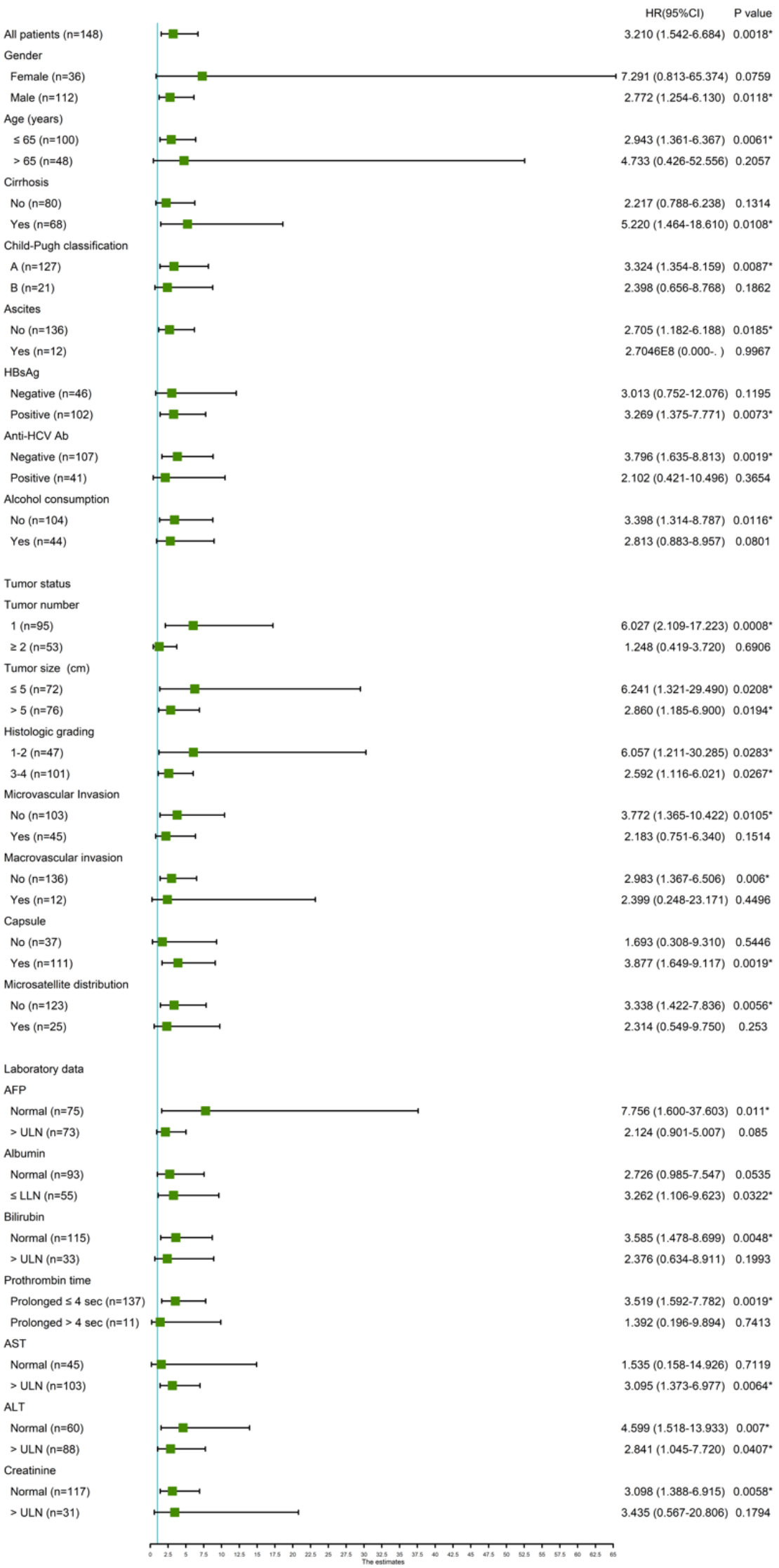

Figure 2. Forest plot of HRs for the associations between high AXNA2 expression and OS in various clinical subgroups. The subgroup-specific HR (95\% CI) is shown by the green box (black lines). Statistically significant differences $(p<0.05)$ are indicated by a single asterisk "**". 
Taken together, these findings suggest that high expression of ANXA2 in HCC cancerous parts could predict shorter OS in HCC patients receiving surgical treatment. However, in patients with more advanced stage of HCC or poorer liver function, non-cirrhosis patients or HBsAg-negative patients, the predictive value diminished.

\section{Discussion}

Previously, ANXA2 was identified as an independent prognostic marker in several cancer types, including laryngeal cancer [16], breast cancer [17], ovarian cancer [18] and endometrial cancer [19]. A similar predictive role of ANXA2 in HCC development has also been reported [9]. In the current study, we found that high expression of ANXA2 in HCC tissues was associated with a significantly shorter OS, indicating that ANXA2 was a predictor for unfavorable prognosis in liver cancer. Carbon tetrachloride $\left(\mathrm{CCl}_{4}\right)$ treatment induces liver fibrosis, which mimics the sequel of chronic virus infection. Longterm $\mathrm{CCl}_{4}$ treatment renders fibrotic liver-to-liver cirrhosis, as a pre-malignant stage of HCC development [20]. Yang and co-workers demonstrated that ANXA2 levels were induced upon $\mathrm{CCl}_{4}$ treatment in Sprague Dawley rats compared to those in the control group [11]. Our results showed that ANXA2 expression was increased in patients with liver cirrhosis compared to those with non-cirrhosis (Table 2). Another report demonstrated that serum ANXA2 levels in chronic hepatitis B patients were significantly higher than those in the normal group [21]. On the other hand, a previous investigation indicated that ANXA2 functioned as a modulator in HCV assembly but not in HCV replication or viron release [22]. Our study revealed that ANXA2 expression was higher in the HCVpositive group compared to the HCV-negative group, suggesting ANXA2 expression was regulated by $\mathrm{HCV}$ infection through a yet unknown mechanism. However, in subgroup analysis, ANXA2 higher expression was not correlated with survival outcome in HCVpositive patients. Taken together, the evidence suggested that ANXA2 may be involved in early-stage HCC development, i.e., liver fibrosis to cirrhosis progression.

In contrast, Liu et al. indicated that ANXA2 expression in serum or HCC tissues were not significantly correlated with survival outcomes [12]. In an Egypt study, ANXA2 expression was lower in cirrhotic group than those in control group in HCC tissues [23]. These controversial results for ANXA2 on survival outcomes of HCC may be explained as follows: First, most of our specimens analyzed in this study were from cirrhotic or HBVrelated patients. Second, in this study, ANXA2 expression in HCC tissues was detected by Western blot analysis followed by densitometry quantification. In contrast, an early study had assayed the serum levels of ANXA2 by ELISA. The detection method (Western blot vs. ELISA) and quantitative criteria may lead to different results. Third, these studies were performed in different countries; thereby, the geographic/ethnic issue may also have caused the inconsistent results. Fourth, a previous study reported that ANXA2 could be secreted to the extracellular environment upon interferon- $\gamma$ treatment [24], suggesting that hepatitis activities might play a role. The intracellular and extracellular ANXA2 proteins also exert different functions [25]. We believe that these are possible reasons for the inconsistencies.

Zhang and co-workers demonstrated that knockdown of ANXA2 in hepatoma cell lines reduced cell migration and invasion [26]. Mechanistically, ANXA2 interacted with CD147 and regulated CD147 localization, thereby inducing matrix metallopoateinase 2 (MMP2) expression. Furthermore, knockdown of ANXA2 in a hepatoma cell line, MHCC97$\mathrm{H}$, repressed cell growth and invasive ability [27]. Oncogenic roles of transgelin-2 in HCC have been demonstrated, and its high expression is associated with ANXA2, which, in turn, promotes tumor metastasis through the NFkB pathway [28]. Another investigation indicated that ANXA2 was involved in immune escape of HCC via modulation of immune cells such as regulatory T cells, natural killer cells and dendritic cells [29]. In addition to HCC, the ANXA2-mediated immunosuppression phenotypes were observed in nasopharyngeal carcinoma cells [30] and renal cell carcinoma [31]. Another study revealed that expression levels of ANXA2 in liver tissues were upregulated in a thioacetamide (TAA)-induced cir- 
rhotic rat model [32]. The authors found that immuno-related factors such as transforming growth factor beta and interleukin were increased in TAA-treated rats, suggesting ANXA2 might be involved in the immune response pathway. A long non-coding RNA, named lung cancer-associated transcript 1 (LUCAT1), induced cell growth and metastasis of hepatoma cell lines in vitro and in vivo [33]. LUCAT1 associated with ANXA2 was identified by an RNA pull-down assay, leading to inhibition of ANXA2 phosphorylation and induction of MMP9 activation. Recently, circular RNA (circRNA) has been found responsible for regulating cancer progression [34]. The expression levels of circ_0021093 were upregulated in HCC specimens, and a higher level of circ_0021093 was correlated with poor survival outcomes [35]. Depletion of circ_0021093 reduced cell proliferation, migration and invasion by modulating miR-432. Moreover, ANXA2 is a direct targeted gene of miR-432. These findings indicated that the circ_0021093/miR-432/ANXA2 axis was another important pathway regulating HCC progression. This evidence supported that ANXA2 played an oncogenic role in liver cancer.

\section{Conclusions}

In conclusion, we showed that ANXA2 was a prognostic marker for HCC patients receiving surgical treatment. However, the predictive value diminished in several clinical subgroups such as those with more advanced stage of HCC or poorer liver function, as well as non-cirrhosis and HBsAg-negative patients.

Supplementary Materials: The following are available online at https://www.mdpi.com/article/10 $.3390 / \mathrm{jcm} 10184158 / \mathrm{s} 1$, Figure S1: Elevated ANXA2 expression was correlated with poor prognosis in patients with HCC in online available dataset analysis.

Author Contributions: Y.-H.L. and C.-T.Y. designed and supervised the study; S.-W.H., Y.-C.C., Y.-H.L. and C.-T.Y. drafted the manuscript; S.-W.H. and Y.-C.C. performed the experiments; S.-W.H., Y.-C.C., Y.-H.L. and C.-T.Y. interpreted the data; S.-W.H., Y.-C.C., Y.-H.L. and C.-T.Y. collected and analyzed the clinical data. All authors have read and agreed to the published version of the manuscript.

Funding: This work was supported by grants from Chang Gung Memorial Hospital, Taiwan (CMRPG3K1551 to YHL).

Institutional Review Board Statement: This study was approved by the Institutional Review Board of Chang Gung Medical Center (IRB: 201900957B0). The experiments conformed to the ethical guidelines of the 1975 Declaration of Helsinki.

Informed Consent Statement: Informed consent was obtained from patients, prior to their participation in the study.

Data Availability Statement: The available datasets can be analyzed and download from Gene Expression Profiling Interactive Analysis (http://gepia.cancer-pku.cn/, accessed on 1 September 2021, Beijing, China) and Gene Expression Omnibus (GEO; http://www.ncbi.nlm.nih.gov/geo, accessed on 1 September 2021, Bethesda MD, USA) with accession numbers GSE14520, respectively.

Acknowledgments: The authors appreciate the technical and administrative support provided by all members of the Liver Research Center in Chang Gung Memorial Hospital, Taoyuan, Taiwan.

Conflicts of Interest: The authors have no conflict to disclose.

\section{References}

1. Kim, E.; Viatour, P. Hepatocellular carcinoma: Old friends and new tricks. Exp. Mol. Med. 2020, 52, 1898-1907. [CrossRef]

2. Elpek, G.O. Molecular pathways in viral hepatitis-associated liver carcinogenesis: An update. World J. Clin. Cases 2021, 9, 4890-4917. [CrossRef]

3. Chiang, C.J.; Yang, Y.W.; You, S.L.; Lai, M.S.; Chen, C.J. Thirty-year outcomes of the national hepatitis B immunization program in Taiwan. JAMA 2013, 310, 974-976. [CrossRef] [PubMed]

4. Park, S.J.; Jang, J.Y.; Jeong, S.W.; Cho, Y.K.; Lee, S.H.; Kim, S.G.; Cha, S.W.; Kim, Y.S.; Cho, Y.D.; Kim, H.S.; et al. Usefulness of AFP, AFP-L3, and PIVKA-II, and their combinations in diagnosing hepatocellular carcinoma. Medicine 2017, 96, e5811. [CrossRef] 
5. Chan, M.Y.; She, W.H.; Dai, W.C.; Tsang, S.H.Y.; Chok, K.S.H.; Chan, A.C.Y.; Fung, J.; Lo, C.M.; Cheung, T.T. Prognostic value of preoperative alpha-fetoprotein (AFP) level in patients receiving curative hepatectomy-An analysis of 1182 patients in Hong Kong. Transl. Gastroenterol. Hepatol. 2019, 4, 52. [CrossRef] [PubMed]

6. Chen, L.; Lin, L.; Xian, N.; Zheng, Z. Annexin A2 regulates glioma cell proliferation through the STAT3cyclin D1 pathway. Oncol. Rep. 2019, 42, 399-413.

7. Lee, D.B.; Jamgotchian, N.; Allen, S.G.; Kan, F.W.; Hale, I.L. Annexin A2 heterotetramer: Role in tight junction assembly. Am. J. Physiol.-Ren. Physiol. 2004, 287, F481-F491. [CrossRef] [PubMed]

8. Jiang, S.L.; Pan, D.Y.; Gu, C.; Qin, H.F.; Zhao, S.H. Annexin A2 silencing enhances apoptosis of human umbilical vein endothelial cells in vitro. Asian Pac. J. Trop. Med. 2015, 8, 952-957. [CrossRef] [PubMed]

9. Sun, Y.; Gao, G.; Cai, J.; Wang, Y.; Qu, X.; He, L.; Liu, F.; Zhang, Y.; Lin, K.; Ma, S.; et al. Annexin A2 is a discriminative serological candidate in early hepatocellular carcinoma. Carcinogenesis 2013, 34, 595-604. [CrossRef] [PubMed]

10. Wang, C.; Guo, Y.; Wang, J.; Min, Z. Annexin A2 knockdown inhibits hepatoma cell growth and sensitizes hepatoma cells to 5-fluorouracil by regulating beta-catenin and cyclin D1 expression. Mol. Med. Rep. 2015, 11, 2147-2152. [CrossRef] [PubMed]

11. Yang, M.; Wang, C.; Li, S.; Xv, X.; She, S.; Ran, X.; Li, S.; Hu, H.; Hu, P.; Zhang, D.; et al. Annexin A2 promotes liver fibrosis by mediating von Willebrand factor secretion. Dig. Liver Dis. 2017, 49, 780-788. [CrossRef]

12. Liu, Z.; Ling, Q.; Wang, J.; Xie, H.; Xu, X.; Zheng, S. Annexin A2 is not a good biomarker for hepatocellular carcinoma in cirrhosis. Oncol. Lett. 2013, 6, 125-129. [CrossRef]

13. Roessler, S.; Jia, H.L.; Budhu, A.; Forgues, M.; Ye, Q.H.; Lee, J.S.; Thorgeirsson, S.S.; Sun, Z.; Tang, Z.Y.; Qin, L.X.; et al. A unique metastasis gene signature enables prediction of tumor relapse in early-stage hepatocellular carcinoma patients. Cancer Res. 2010, 70, 10202-10212. [CrossRef] [PubMed]

14. Lin, Y.H.; Wu, M.H.; Liu, Y.C.; Lyu, P.C.; Yeh, C.T.; Lin, K.H. LINC01348 suppresses hepatocellular carcinoma metastasis through inhibition of SF3B3-mediated EZH2 pre-mRNA splicing. Oncogene 2021, 40, 4675-4685. [CrossRef] [PubMed]

15. Mazumdar, M.; Glassman, J.R. Categorizing a prognostic variable: Review of methods, code for easy implementation and applications to decision-making about cancer treatments. Stat. Med. 2000, 19, 113-132. [CrossRef]

16. Luo, S.; Xie, C.; Wu, P.; He, J.; Tang, Y.; Xu, J.; Zhao, S. Annexin A2 is an independent prognostic biomarker for evaluating the malignant progression of laryngeal cancer. Exp. Ther. Med. 2017, 14, 6113-6118. [CrossRef]

17. Zhang, F.; Zhang, H.; Wang, Z.; Yu, M.; Tian, R.; Ji, W.; Yang, Y.; Niu, R. P-glycoprotein associates with Anxa2 and promotes invasion in multidrug resistant breast cancer cells. Biochem. Pharm. 2014, 87, 292-302. [CrossRef]

18. Lokman, N.A.; Pyragius, C.E.; Ruszkiewicz, A.; Oehler, M.K.; Ricciardelli, C. Annexin A2 and S100A10 are independent predictors of serous ovarian cancer outcome. Transl. Res. 2016, 171, 83-95.e2. [CrossRef]

19. Alonso-Alconada, L.; Santacana, M.; Garcia-Sanz, P.; Muinelo-Romay, L.; Colas, E.; Mirantes, C.; Monge, M.; Cueva, J.; Oliva, E.; Soslow, R.A.; et al. Annexin-A2 as predictor biomarker of recurrent disease in endometrial cancer. Int. J. Cancer 2015, 136, 1863-1873. [CrossRef]

20. Scholten, D.; Trebicka, J.; Liedtke, C.; Weiskirchen, R. The carbon tetrachloride model in mice. Lab. Anim. 2015, 49 (Suppl. 1), 4-11. [CrossRef]

21. Kolgelier, S.; Demir, N.A.; Inkaya, A.C.; Sumer, S.; Ozcimen, S.; Demir, L.S.; Pehlivan, F.S.; Arslan, M.; Arpaci, A. Serum Levels of Annexin A2 as a Candidate Biomarker for Hepatic Fibrosis in Patients with Chronic Hepatitis B. Hepat. Mon. 2015, 15, e30655. [CrossRef]

22. Backes, P.; Quinkert, D.; Reiss, S.; Binder, M.; Zayas, M.; Rescher, U.; Gerke, V.; Bartenschlager, R.; Lohmann, V. Role of annexin A2 in the production of infectious hepatitis C virus particles. J. Virol. 2010, 84, 5775-5789. [CrossRef] [PubMed]

23. Shaker, M.K.; Abdel Fattah, H.I.; Sabbour, G.S.; Montasser, I.F.; Abdelhakam, S.M.; El Hadidy, E.; Yousry, R.; El Dorry, A.K. Annexin A2 as a biomarker for hepatocellular carcinoma in Egyptian patients. World J. Hepatol. 2017, 9, 469-476. [CrossRef] [PubMed]

24. Chen, Y.D.; Fang, Y.T.; Cheng, Y.L.; Lin, C.F.; Hsu, L.J.; Wang, S.Y.; Anderson, R.; Chang, C.P.; Lin, Y.S. Exophagy of annexin A2 via RAB11, RAB8A and RAB27A in IFN-gamma-stimulated lung epithelial cells. Sci. Rep. 2017, 7, 5676. [CrossRef]

25. Hitchcock, J.K.; Katz, A.A.; Schafer, G. Dynamic reciprocity: The role of annexin A2 in tissue integrity. J. Cell Commun. Signal. 2014, 8, 125-133. [CrossRef]

26. Zhang, W.; Zhao, P.; Xu, X.L.; Cai, L.; Song, Z.S.; Cao, D.Y.; Tao, K.S.; Zhou, W.P.; Chen, Z.N.; Dou, K.F. Annexin A2 promotes the migration and invasion of human hepatocellular carcinoma cells in vitro by regulating the shedding of CD147-harboring microvesicles from tumor cells. PLoS ONE 2013, 8, e67268.

27. Dong, Z.; Yao, M.; Zhang, H.; Wang, L.; Huang, H.; Yan, M.; Wu, W.; Yao, D. Inhibition of Annexin A2 gene transcription is a promising molecular target for hepatoma cell proliferation and metastasis. Oncol. Lett. 2014, 7, 28-34. [CrossRef]

28. Shi, J.; Ren, M.; She, X.; Zhang, Z.; Zhao, Y.; Han, Y.; Lu, D.; Lyu, L. Transgelin-2 contributes to proliferation and progression of hepatocellular carcinoma via regulating Annexin A2. Biochem. Biophys. Res. Commun. 2020, 523, 632-638. [CrossRef]

29. Qiu, L.W.; Liu, Y.F.; Cao, X.Q.; Wang, Y.; Cui, X.H.; Ye, X.; Huang, S.W.; Xie, H.J.; Zhang, H.J. Annexin A2 promotion of hepatocellular carcinoma tumorigenesis via the immune microenvironment. World J. Gastroenterol. 2020, 26, 2126-2137. [CrossRef]

30. Chen, C.Y.; Lin, Y.S.; Chen, C.H.; Chen, Y.J. Annexin A2-mediated cancer progression and therapeutic resistance in nasopharyngeal carcinoma. J. Biomed. Sci. 2018, 25, 30. [CrossRef] [PubMed] 
31. Aarli, A.; Skeie Jensen, T.; Kristoffersen, E.K.; Bakke, A.; Ulvestad, E. Inhibition of phytohaemagglutinin-induced lymphoproliferation by soluble annexin II in sera from patients with renal cell carcinoma. APMIS 1997, 105, 699-704. [CrossRef] [PubMed]

32. An, J.H.; Seong, J.; Oh, H.; Kim, W.; Han, K.H.; Paik, Y.H. Protein expression profiles in a rat cirrhotic model induced by thioacetamide. Korean J. Hepatol. 2006, 12, 93-102. [PubMed]

33. Lou, Y.; Yu, Y.; Xu, X.; Zhou, S.; Shen, H.; Fan, T.; Wu, D.; Yin, J.; Li, G. Long non-coding RNA LUCAT1 promotes tumourigenesis by inhibiting ANXA2 phosphorylation in hepatocellular carcinoma. J. Cell. Mol. Med. 2019, 23, 1873-1884. [CrossRef]

34. Cheng, D.; Wang, J.; Dong, Z.; Li, X. Cancer-related circular RNA: Diverse biological functions. Cancer Cell Int. 2021, 21, 11. [CrossRef] [PubMed]

35. Wang, Y.; Xu, W.; Zu, M.; Xu, H. Circular RNA circ_0021093 regulates miR-432/Annexin A2 pathway to promote hepatocellular carcinoma progression. Anticancer Drugs 2021, 32, 484-495. [CrossRef] 